\title{
EDUCACIÓN MÉDICA EN COLOMBIA Estado del arte y perspectivas
}

Pablo Alberto Galvis Centurión, MD *

\section{Resumen}

La educación superior en Colombia se encuentra en la actualidad en un punto coyuntural de grandes cambios que beneficiarán en forma directa a los estudiantes, los docentes y a la sociedad. La formación médica no es ajena a estos cambios y para fortuna nuestra se plantea ahora un nuevo modelo donde el punto de partida es el alumno, lo que ha permitido buscar otras estrategias cuyo planteamiento es que el trabajo académico no debe reducirse a escuchar las explicaciones del docente o hacer lo que éste señala. La acción del profesor de hoy es más de orientador y facilitador del conocimiento, no un transmisor del mismo para que el alumno tenga una actitud activa y trabaje en la construcción de sus propios conocimientos, de tal manera que aprenda y comprenda los conceptos adquiridos.

Para el análisis adecuado del tema de la educación superior en Colombia debemos entender el concepto de universidad como una comunidad académica ${ }^{1}$ y una organización social ${ }^{2}$ que produce, transmite y se apropia del conocimiento, entendido éste como ciencia por lo que forma al ser humano por la vida y para la vida de una manera integral . La universidad se sustenta en cuatro parámetros, que son: ser científica, universal, corporativa y autónoma; además, tiene que realizar tres tareas: investigación, docencia y extensión. ${ }^{3}$

La docencia universitaria en el área de las ciencias es muy diferente a la formación universitaria de otras áreas del conocimiento y específicamente en lo referente a la medicina se encuentran las más importantes discrepancias.

El médico representa un ser social de gran relevancia para muchas comunidades y desde este punto de vista no sólo son importantes sus conocimientos en salud, sino que toma mayor interés su formación integral, por lo cual en nuestras instituciones educativas de nivel superior se hace necesario implementar cambios que suplan las necesidades sociales y sobrepasen las expectativas de la comunidad científica.

Jefe de residentes de gineco-obstetricia, Hospital de San José. Fundación Universitaria de Ciencias de la Salud. Diplomado docencia universitaria con énfasis en salud, FUCS. Docente núcleo electivo. Facultad de Medicina, FUCS.
A nivel mundial se ha ido produciendo conciencia de la inadecuada atención de la salud que se brinda hoy en día, en especial en los países subdesarrollados como los latinoamericanos; la educación médica debe responder, en lo que le corresponde, a esta necesidad.

En América Latina la Organización Panamericana de la Salud y el proyecto EMA (Educación Médica para América Latina), han desarrollado intensas actividades para que la educación explore más allá de los cambios cosméticos y adecúe su quehacer a las necesidades subregionales. Solo así se podrá responder a los principios elementales de la pedagogía moderna del adulto. ${ }^{4}$ Todo esto se generó a partir de las reuniones internacionales en Dinamarca organizadas por World Federation for Medical Education en 1999 y la fundación el mismo año del Instituto para la educación médica internacional en Nueva York, el cual mediante un comité especializado desarrolló el concepto de requisitos globales mínimos esenciales y definió un conjunto de resultados mínimos del aprendizaje que los estudiantes de medicina deben demostrar para obtener su grado. Y por último, en octubre de 2001 se publicó la Declaration of Granada on standars for Undergraduate Medical Education que contribuye también a formular las estrategias del cambio radical en la enseñanza medica. ${ }^{5}$ 
En la actualidad el proceso de cambio y renovación educacional es muy fuerte y tiene un carácter global, por lo cual nuestro país no puede ser ajeno a esta tendencia. El proceso ha ido demostrando que la educación de adultos debe centrarse en el estudiante y los métodos tradicionales deben dejar el lugar a otros más productivos; de tal forma, prestigiosas universidades como la Nacional de Colombia han implementado un concurso de méritos para proveer 300 cargos docentes a los jóvenes colombianos, logrando no solo un cambio generacional en la docencia, sino una oportunidad para herramientas pedagógicas de actualidad.

\section{Situación actual de la enseñanza médica en América Latina y Colombia}

A pesar de que ya existen serios intentos de innovación en varios países, no deja de ser inquietante el futuro de este proceso; se requiere trabajar con las generaciones futuras de profesionales de la salud, sin olvidar los médicos titulados en ejercicio, los cuales ya superan 1'000.000 en nuestro continente.

La función de construir conocimientos e incidir en la formación integral de las personas no es una prioridad de la universidad. La mayor parte de los centros de educación superior carecen de programas de estudios pedagógicos para la formación de sus docentes y consideran que el conocimiento científico es suficiente y los capacita para enseñar, ignorando la formación pedagógica, requisito básico para mejorar la educación. No es raro que grandes sabios de la ciencia, con innumerables publicaciones internacionales, no logran transmitir sus conocimientos a otros por la falta de argumentos pedagógicos para tal fin. En otras palabras, se incumple el articulo 68 de la constitución política de Colombia "la enseñanza estará a cargo de personas de reconocida idoneidad ética y pedagógica. La ley garantiza la profesionalización y dignificación de la actividad docente". ${ }^{3}$ En Colombia, según estadisticas del ICFES, el 50\% de los profesores solo tienen nivel de pregrado, $18 \%$ de especialización, $14 \%$ de maestría y solo un $2 \%$ de doctorado.
En la educación superior la tarea de producir y crear conocimientos es muy escasa; en Colombia tan solo se cuenta con alrededor de 3.000 científicos, de los cuales la mitad no ha realizado estudios de maestría o doctorado. Paises industrializados como Japón cuentan con 4.800 cientificos por cada millón de habitantes y E.U.A. entre 2.600 y 3.200 ; para un nivel adecuado de competencia. Colombia debería tener en la actualidad según su poblacion, cerca de 38.000 cientificos. Solo $1 \%$ de los del mundo son latinoamericanos y de estos $1 \%$ colombianos.

A la universidad le compete realizar investigaciones que constituyan un verdadero aporte al desarrollo de las ciencias, además de crear nuevas tecnologías y aportar soluciones a los problemas del país. ${ }^{3}$

Es probable que el más importante en la actualidad sea la calidad de la educación superior y de la enseñanza médica en nuestro país. La universidad es en esencia repetidora y distribuidora de conocimientos, antes que creadora de los mismos. Es demasiado académica y teórica, se le enseña al estudiante a responder conceptos antes que a pensar por sí mismo, sólo brinda conocimientos para que sus alumnos puedan ejercer una profesión, olvidando el componente humanista y la formación para la vida.

De acuerdo con la Ley 30 de 1992, las universidades son autónomas para crear programas, emitir reglamentos y nombrar sus autoridades, lo cual ha permitido crear cerca de 3.000 nuevos programas sin que exista un claro control sobre las necesidades y la calidad de los mismos, llevando a que muchas instituciones vean al estudiante como un cliente muy rentable.

Se ha estudiado y analizado la situación actual de la educación médica en latinoamérica en múltiples escenarios desde mediados de la década de los noventa. En nuestro país se realizó la tercera conferencia mundial sobre educación médica (Bogotá, octubre de 1995) donde se demostró un alto interés y decisión para trabajar hacia los cambios educacionales en las ciencias de la salud; después en otras regiones del globo terráqueo, como España, Canadá, Dinamarca, EUA, 
Chile y Cuba, se han efectuado diversas reuniones que permiten generar una propuesta innovadora. Hoy en día se plantean diversas alternativas de cambio, muchas de las cuales ya se vienen implementando en algunas universidades y que vislumbran mejores resultados en la calidad docente.

\section{El consenso y las conclusiones básicas de la educación médica moderna}

Los puntos fundamentales de consenso sobre la dirección que debe tomar la educación médica han ido apareciendo en forma cada vez más consistente. El modelo innovador centra la educación en el estudiante, se basa en problemas, es integrado, gira alrededor de la medicina comunitaria sin excluir la hospitalaria, favorece los núcleos electivos y asegura un aprendizaje sistemático. Los principales ítems de esta nueva propuesta son los siguientes:-4

1. Educación centrada en el estudiante.

2. Educación integrada e integradora.

3. Aprendizaje basado en problemas.

4. Relevancia de los problemas prioritarios.

5. Evaluación formativa vs. sumativa.

6. Núcleos de contexto y electivos.

7. Igual énfasis en conocimientos, destrezas y actitudes.

8. Selección de conocimientos esenciales.

9. Favorecer la formación de los docentes más allá de su especialidad.

10.Estrechar los lazos entre docentes y los estudiantes.
11. Desarrollar la capacidad de análisis y de evaluación crítica.

12. Actuar en grupos pequeños y con docentes facilitadores. $^{4}$

El cuerpo de conocimientos de la medicina es enorme y creciente. Hoy no puede ser adquirido por una persona en su totalidad, como sí lo fué a comienzos del siglo $\mathrm{XX}$, cuando la medicina era un mero oficio. Por consiguiente, las facultades de medicina deben concebir planes de estudio flexibles y versátiles que hagan posible la emergencia de un producto diversificado, diferente y adicional al uniforme que en la actualidad se denomina médico general. ${ }^{6}$

El camino inicial para lograr los objetivos de una educación médica futurista es la evaluación del proceso tradicional empleado en nuestras instituciones, con la comprensible resistencia al cambio que esto genera. De otra forma, no lograremos actualizar a nuestros estudiantes en la medicina de hoy ni permitirles por consiguiente ejercer mañana su profesión de una manera idónea y ética, resolviendo las necesidades de su comunidad.

\section{Referencias}

1. Ricardo L. ¿Qué tan Universidad es Nuestra Universidad? Educación y Cultura. Bogotá: Fecode; 1992. pp.55-61 (Educación y Cultura no.26, Centro de Estudios y de Investigaciones Docentes (CEID).

2. Schumacher Ch. Universidad y la Cultura del Conocimiento. Rev. Cole Mayor Ntra. Sra. Rosario 1998; 91 (581): 74-81.

3. Rojas Soto E. H. Educación Superior en Colombia. Reflexiones sobre Educación Universitaria, Bogotá: Universidad Nacional de Colombia, Grupo de Apoyo Pedagógico y Formación Docente, 2003 pp 25-36.

4. Venturelli J. Educación Médica - Nuevos Enfoques Metas y Métodos. Washington: OPS, 2003 pp 2-32 (Serie Paltex Salud y Sociedad 2000).

5. Declaration of Granada on Standard for Undergraduate Medical Education, 24 th Edu. Méd 2002, 5 (1)

6. Patiño Restrepo J F. La Formación del Médico. (citado 2004 abr) Disponible en www.FEPAFEM.com

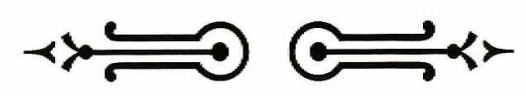

